Bundesgesundheitsbl 2022 $\cdot$ 65:202-208 https://doi.org/10.1007/s00103-021-03476-w Eingegangen: 2. August 2021

Angenommen: 7. Dezember 2021 Online publiziert: 4. Januar 2022 (c) Der/die Autor(en) 2021

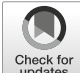

Reimar Johne - Nadine Althof · Karsten Nöckler · Alexander Falkenhagen

Bundesinstitut für Risikobewertung, Berlin, Deutschland

\title{
Das Hepatitis-E-Virus - ein zoonotisches Virus: Verbreitung, Übertragungswege und Bedeutung für die Lebensmittelsicherheit
}

Vorhandensein besserer diagnostischer Tests zurückgeführt. Neben diesen klinisch auffälligen Erkrankungsverläufen scheint eine HEV-Infektion jedoch auch häufig mit nur milden Symptomen einherzugehen oder sogar asymptomatisch zubleiben. Serologische Untersuchungen zeigen, dass im Jahr 2010 etwa 15,3\% der deutschen Bevölkerung HEV-spezifische IgG-Antikörper besaßen, die auf eine vergangene Infektion hinweisen [3].

Das HEV ist ein etwa 30-40 nm großes Viruspartikel, das ein Genom aus Einzelstrang-RNA mit positiver Polarität besitzt. Während es über den Darmtrakt als unbehülltes Virus ausgeschieden wird, findet man im Serum von Patienten und im Überstand infizierter Zellkulturen vor allem Partikel, die eine zusätzliche Lipidhülle besitzen. HEV wird in die Familie Hepeviridae, Genus Orthohepevirus eingeordnet, in dem Stämme der Spezies Orthohepevirus A für den Menschen die wichtigste Rolle spielen (• Tab. 1). Innerhalb dieser Spezies wurden bisher 8 unterschiedliche Genotypen beschrieben, die sich bezüglich ihres Wirtsspektrums und ihrer Übertragungswege teilweise deutlich unterscheiden [4].

Die Genotypen 1 und 2 infizieren ausschließlich den Menschen und werden vor allem über kontaminiertes Trinkwasser übertragen. Dies führt in Entwicklungsländern häufig zu großen Krankheitsausbrüchen. Bei der Infektion von Schwangeren sind auch vertikale Übertragungen dieser Genotypen möglich
[4]. Demgegenüber sind die Genotypen 3 und 4 zoonotisch und haben ihr Hauptreservoir in Haus- und Wildschwein, von denen sie vor allem über den Verzehr von Fleischprodukten auf den Menschen übertragen werden können. Diese Genotypen sind vorrangig in Industrieländern weitverbreitet und führen hier zu sporadischen Hepatitis-E-Fällen. Neben dem zoonotischen Übertragungsweg können diese Genotypen auch parenteral über Blut und Blutprodukte übertragen werden [4]. Aus der Virusspezies Orthohepevirus A wurde weiterhin der Genotyp 7 beim Menschen gefunden, der sein Reservoir vor allem in Dromedaren hat und im Mittleren Osten vorkommt. Als weiteres humanpathogenes Virus wurde kürzlich das Ratten-HEV identifiziert, das besonders in Ratten verbreitet ist und zur Spezies Orthohepevirus $C$ gehört $[1,4]$. - Tab. 1 zeigt einen Überblick über die Hepevirusspezies, Genotypen und deren wichtigste Charakteristika.

In Deutschland und in vielen anderen europäischen Ländern werden hauptsächlich Infektionen mit dem HEVGenotyp 3 mit den Subtypen 3c, 3e und $3 f$ gefunden [5]. Diese Subtypen werden hier gleichermaßen auch in Schweinen, Wildschweinen und anderen Tierarten nachgewiesen, weshalb ein zoonotischer Übertragungsweg auf den Menschen über den Kontakt mit den Tieren oder über den Verzehr von Fleischprodukten wahrscheinlich ist. In 


\begin{tabular}{|c|c|c|c|c|}
\hline Spezies & $\begin{array}{l}\text { Geno- } \\
\text { typ }\end{array}$ & Hauptsächlicher Wirt & $\begin{array}{l}\text { Hauptsächliche geografische } \\
\text { Verbreitung }\end{array}$ & $\begin{array}{l}\text { Hauptsächlicher Übertragungsweg } \\
\text { zum Menschen }\end{array}$ \\
\hline \multirow{8}{*}{$\begin{array}{l}\text { Orthohepe- } \\
\text { virus } A\end{array}$} & 1 & Mensch & Asien, Afrika & Verunreinigtes Trinkwasser \\
\hline & 2 & Mensch & Mittelamerika, Afrika & Verunreinigtes Trinkwasser \\
\hline & 3 & Mensch, Schwein, Wildschwein & Weltweit & Verzehr von Fleischprodukten \\
\hline & 4 & Mensch, Schwein, Wildschwein & Südostasien & Verzehr von Fleischprodukten \\
\hline & 5 & Wildschwein & Japan & - \\
\hline & 6 & Wildschwein & Japan & - \\
\hline & 7 & Dromedar, Mensch & Mittlerer Osten & Unklar \\
\hline & 8 & Trampeltier & Südostasien & - \\
\hline $\begin{array}{l}\text { Orthohepe- } \\
\text { virus } B\end{array}$ & n.d. & Verschiedene Vogelarten & Weltweit & - \\
\hline $\begin{array}{l}\text { Orthohepe- } \\
\text { virus C }\end{array}$ & n.d. & $\begin{array}{l}\text { Verschiedene Rattenarten, Frettchen, Nerz, } \\
\text { Mensch }\end{array}$ & Weltweit & Unklar \\
\hline $\begin{array}{l}\text { Orthohepe- } \\
\text { virus D }\end{array}$ & n.d. & Verschiedene Fledermausarten & Weltweit & - \\
\hline \multicolumn{5}{|c|}{ n. $d$. nicht definiert, - keine Zoonose } \\
\hline
\end{tabular}

Tab. 2 Häufigkeit von HEV-Infektionen in Wild- und Hausschweinen in Deutschland

\begin{tabular}{|l|lll|}
\hline Tierart & $\begin{array}{l}\text { Nachweis von HEV-RNA } \\
\text { (Zahl positiver/Gesamtzahl un- } \\
\text { tersuchter Tiere) }\end{array}$ & $\begin{array}{l}\text { Nachweis HEV-spezifischer Antikör- } \\
\text { per } \\
\text { (Zahl positiver/Gesamtzahl unter- } \\
\text { suchter Tiere) }\end{array}$ & $\begin{array}{l}\text { Refe- } \\
\text { renz }\end{array}$ \\
\hline $\begin{array}{l}\text { Haus- } \\
\text { schwein }\end{array}$ & - & $534 / 1072(49,8 \%)$ & {$[38]$} \\
& - & $354 / 516(68,6 \%)$ & {$[39]$} \\
& $34 / 251(13,5 \%)$ & - & {$[40]$} \\
& - & $1065 / 2273(46,9 \%)$ & {$[41]$} \\
\hline & - & $187 / 438(42,7 \%)$ & {$[42]$} \\
\hline Wild- & $1 / 105(1,0 \%)$ & - & {$[35]$} \\
schwein & $3 / 120(2,5 \%)$ & - & {$[43]$} \\
& $43 / 259(17,2 \%)$ & $155 / 250(62,0 \%)$ & {$[44]$} \\
\hline $17 / 189(5,3 \%)$ & - & {$[32]$} \\
\hline $22 / 148(14,9 \%)$ & - & {$[7]$} \\
\hline $48 / 126(38,1 \%)$ & $32 / 107(29,9 \%)$ & {$[33]$} \\
\hline & - & $109 / 330(33,0 \%)$ & {$[34]$} \\
& $18 / 124(14,5 \%)$ & - & {$[35]$} \\
\hline $14 / 134(10,4 \%)$ & - & {$[36]$} \\
\hline $39 / 232(16,8 \%)$ & $81 / 180(45,0 \%)$ & {$[37]$} \\
\hline $4 / 104(3,8 \%)$ & $12 / 104(11,5 \%)$ & \\
\hline
\end{tabular}

diesem Artikel sollen die Übertragungswege des HEV in Deutschland und die daraus resultierenden Konsequenzen für die Lebensmittelsicherheit genauer betrachtet werden. Hierzu wird zunächst die aktuelle Literatur zur Verbreitung des Virus - im Speziellen des Genotyps 3 - in Tieren, zu prinzipiell möglichen Übertragungswegen und zu Untersuchungen an Lebensmitteln vorgestellt, um anschließend sowohl Schlussfolgerungen für die Verhinderung einer Virusüber- tragung durch Lebensmittel zu ziehen als auch Forschungsbedarf zur Klärung offener Fragen aufzuzeigen.

\section{Verbreitung des HEV- Genotyps 3 in Tieren}

Zur Verbreitung von HEV-Infektionen in Hausschweinen liegen weltweit zahlreiche Studien vor [6]. Hierbei sind Studien zur HEV-Antikörperprävalenz von solchen zur HEV-RNA-Prävalenz zu unterscheiden. Während Antikörper eine durchgemachte Infektion anzeigen und damit auch weit zurückliegende Infektionen nachweisen können, deuten Virus-RNA-Nachweise auf eine Infektion zum Untersuchungszeitpunkt hin. - Tab. 2 fasst die Ergebnisse von Studien zur HEV-Antikörper- und HEV-RNAPrävalenz in Haus- und Wildschweinen in Deutschland zusammen. Hierbei zeigen sich bei Hausschweinen Antikörperprävalenzen von 42,7-68,6\%, während die Virus-RNA-Prävalenzen zum Zeitpunkt der Schlachtung zwischen $1,0 \%$ und $17,2 \%$ liegen. Die im Vergleich zur Antikörperprävalenz niedrige Virus-RNA-Prävalenz zum Zeitpunkt der Schlachtung spiegelt die Tatsache wider, dass die HEV-Infektion beim Hausschwein vor allem in den ersten Lebensmonaten stattfindet, während $\mathrm{zu}$ späteren Zeitpunkten das Virus oft bereits aus dem Körper eliminiert worden ist.

Auch Wildschweine wurden in zahlreichen Studien auf HEV-Infektionen untersucht (•Tab. 2). Hierbei sind für Deutschland Antikörperprävalenzen von $11,5-45,0 \%$ und RNA-Prävalenzen von $3,8-38,1 \%$ berichtet worden. Die hier stärker zutage tretenden Schwankungen lassen sich vor allem mit Unterschieden in verschiedenen Jagdgebieten erklären, wobei auch nah beieinanderliegende Gebiete große Unterschiede in den Nachweisraten aufweisen können 
Bundesgesundheitsbl 2022 -65:202-208 https://doi.org/10.1007/s00103-021-03476-w

(c) Der/die Autor(en) 2021

\section{R. Johne $\cdot$ N. Althof $\cdot$ K. Nöckler $\cdot$ A. Falkenhagen}

\section{Das Hepatitis-E-Virus - ein zoonotisches Virus: Verbreitung, Übertragungswege und Bedeutung für die Lebensmittelsicherheit}

\section{Zusammenfassung}

Das Hepatitis-E-Virus (HEV) ist ein Erreger einer akuten Hepatitis beim Menschen. Darüber hinaus treten zunehmend auch chronische Infektionen mit fataler Leberzirrhose bei immunsupprimierten Transplantationspatienten auf. Die Zahl der gemeldeten Hepatitis-EFälle in Deutschland hat in den vergangenen Jahren stark zugenommen. Hier kommt vor allem der Genotyp 3 vor, der zoonotisch von Tieren auf den Menschen übertragen werden kann. Haus- und Wildschweine, die ohne die Ausbildung klinischer Symptome infiziert werden, stellen das Hauptreservoir dar. In diesem Artikel werden die Verbreitung von HEV in Tieren in Deutschland, mögliche Übertragungswege des Virus und insbe- sondere die Bedeutung von Lebensmitteln bei der Übertragung anhand der aktuellen wissenschaftlichen Literatur dargestellt. HEV ist in Haus- und Wildschweinen in Deutschland stark verbreitet und wird hauptsächlich über direkten Kontakt oder den Verzehr von Lebensmitteln, die aus diesen Tieren hergestellt wurden, auf den Menschen übertragen. Beim HEV-RNANachweis in spezifischen Lebensmitteln bleibt allerdings oft unklar, ob das enthaltene Virus noch infektiös ist oder durch die Herstellungsbedingungen inaktiviert wurde. Neuere Studien weisen auf eine hohe Stabilität des HEV unter verschiedenen physikochemischen Bedingungen hin, wohingegen eine
Inaktivierung unter anderem durch Erhitzung erreicht wird. Generell wird deshalb ein ausreichendes Erhitzen von Schweinefleisch und -leber vor dem Verzehr empfohlen und für Risikogruppen zusätzlich der Verzicht auf den Verzehr kurzgereifter Rohwürste. Weitere Forschungen sind nötig, um relevante Risikolebensmittel zu identifizieren, alternative Übertragungswege zu untersuchen und effiziente Maßnahmen zu entwickeln, die eine zoonotische Virusübertragung zukünftig verringern oder vermeiden.

\section{Schlüsselwörter}

Hepatitis E - Zoonose - Schwein - Fleischprodukte - Virus-Inaktivierung

\section{Hepatitis E virus-a zoonotic virus: distribution, transmission pathways, and relevance for food safety}

\section{Abstract}

The hepatitis $E$ virus (HEV) is an etiological agent of acute hepatitis in humans. In addition, chronic infections resulting in fatal liver cirrhosis currently emerge in immunosuppressed transplant patients. The number of notified hepatitis $E$ cases in Germany has steeply increased in recent years. Here, genotype 3 , which can be zoonotically transmitted from animals to humans, is predominant. The main reservoirs are pigs and wild boars, which show no signs of infection. In this article, the distribution of HEV in animals in Germany, possible transmission pathways, and especially the importance of food as a transmission vehicle are presented based on the current scientific literature. $\mathrm{HEV}$ is widely spread among domestic pigs and wild boars in Germany and the virus is mainly transmitted by direct contact or by consumption of food produced from those animals. However, if HEV RNA is detected in specific food it is often unclear whether the contained virus is still infectious or inactivated by the conditions during production. Recent studies indicate a high stability of HEV against different physicochemical conditions, whereas - among others - the virus can be efficiently inactivated by heating. Therefore, proper heating of pork meat and liver prior to consumption in general is recommended. For risk groups, avoiding shortly cured raw sausages is an additional suggestion. Further research is necessary to identify relevant risk food products, to investigate alternative transmission pathways, and to develop efficient measures in order to reduce or prevent zoonotic transmissions of the virus in future.

\section{Keywords}

Hepatitis E · Zoonosis · Pig · Meat products . Virus inactivation
[7, 8]. Ein weiterer Unterschied zur Infektion beim Hausschwein scheint die breitere Verteilung von RNA-positiven Tieren in verschiedenen Altersgruppen zu sein. Auch ältere Tiere tragen hier häufiger HEV-RNA. Dies könnte einerseits mit unterschiedlichen Übertragungsmustern bei den frei laufenden Wildschweinen im Vergleich $\mathrm{zu}$ den im Stall gehaltenen Hausschweinen erklärt werden. Andererseits gibt es auch Hinweise, dass Wildschweine eventuell häufiger chronische HEV-Infektionen ausbilden können.

Verschiedene Studien liefern Hinweise auf ein breites Spektrum von emp- fänglichen Tierarten [9]. Kaninchen zeigen hohe Nachweisraten, die auf Infektionen mit einem speziellen Kaninchensubtyp innerhalb des Genotyps 3 zurückzuführen sind. Vereinzelte humane Fälle von Infektionen mit diesem Genotyp weisen das zoonotische Potenzial dieser Virusvariante nach. In den meisten anderen Fällen werden dagegen nur niedrige Nachweisraten sowohl bei Antikörpern als auch bei HEV-RNA gefunden, was gegen eine wichtige Funktion dieser Tierarten als Virusreservoir für humane Infektionen spricht. Vergleichsweise häufig wurden HEV-RNA-Nachweise hingegen bei Wildwiederkäuern berich- tet. Eine Studie in Deutschland erbrachte RNA-Prävalenzen von 6,4\% für Rehwild und 2,4\% für Rotwild [10]. Da positive Nachweise in Wildwiederkäuern vor allem bei gleichzeitig hohen HEV-Prävalenzen in Wildschweinen desselben Gebietes gefunden werden und die detektierten Stämme oft identisch sind, wird hier von „Spillover-Infektionen“ ausgegangen. Für andere Tierarten in Deutschland liegen nur wenige Daten vor. Eine Studie, deren Fokus auf Rindern lag, erbrachte nur negative Resultate [11]. Eine detaillierte Studie an Zootieren zeigte, dass vor allem Schweineartige und Fleischfresser hohe Antikörperprävalen- 


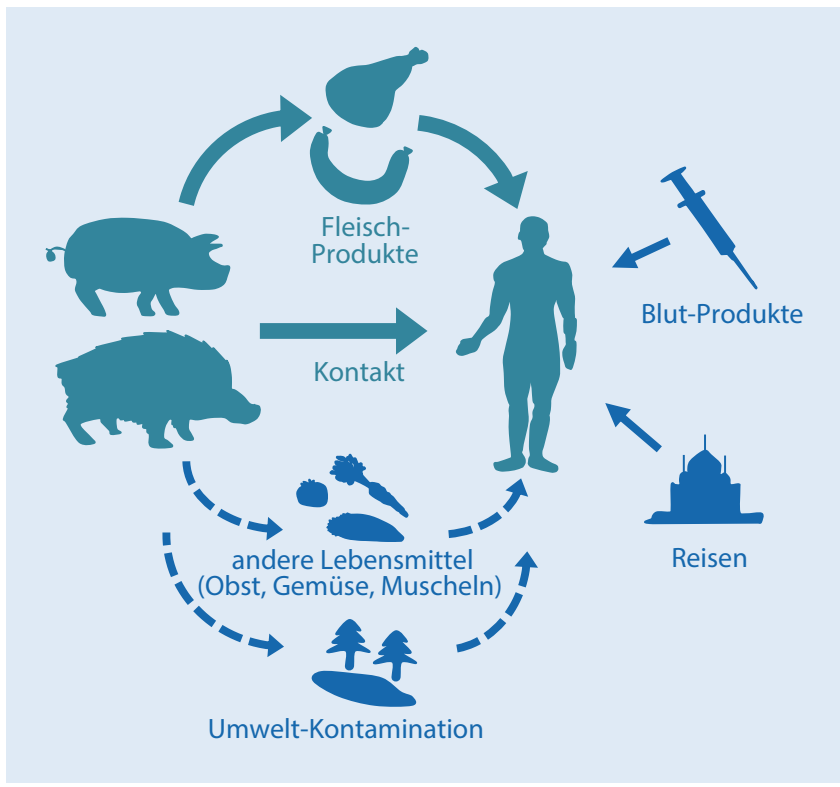

Abb. $1 \varangle$ Mögliche Übertragungswege von HEV in Deutschland. Die Hauptübertragungswege (türkis) sind: direkter Kontakt zu Hausschweinen und Wildschweinen sowie Verzehr von entsprechenden Fleischprodukten. Dunkelblau: weitere Übertragungswege; gestrichelte Pfeile: bisher wenig untersuchte Übertragungswege. (Quelle: eigene Abbildung) zen aufweisen [12]. Bei den Fleischfressern ist allerdings noch unklar, ob sie auch auf den Menschen übertragbare HEVTypen beherbergen, bei ihnen werden vor allem spezielle Stämme aus der Spezies Orthohepevirus $C$ gefunden.

\section{Übertragungswege des HEV- Genotyps 3}

Beim HEV-Genotyp 3 sind verschiedene Übertragungswege bekannt (• Abb. 1). Aufgrund der weiten Verbreitung des Virus in Tierreservoiren wurde in verschiedenen Studien unter anderem die Übertragung über direkten Kontakt zu Tieren untersucht. Dies betrifft hauptsächlich serologische Studien, in denen die Antikörperprävalenz von beruflich mit Tieren in Kontakt Stehenden mit der einer Kontrollgruppe verglichen wurde. In den meisten Fällen weisen die Ergebnisse auf ein höheres Infektionsrisiko der tierexponierten Gruppe hin, so beispielsweise bei Wildschweinjägern in Japan mit einer Antikörperprävalenz von 25,5\% im Gegensatz zu 5,5\% bei nichtjagenden Bewohnern derselben Region oder von $30 \%$ bei Arbeitern auf Schweinefarmen in Norwegen im Vergleich zu $14 \%$ bei Blutspendern [6]. Eine deutsche Studie fand eine Antikörperprävalenz von $28,3 \%$ in der Gruppe von beruflich mit Schweinen Arbeitenden im Vergleich zu 15,5\% bei Blutspendern [13].
Eine andere Studie fand bei Waldarbeitern in Deutschland eine Antikörperprävalenz von $18 \%$ im Vergleich zu $11 \%$ bei Blutspendern [14]. Auch wenn hier der konkrete Übertragungsweg unklar ist, könnte der erhöhte Kontakt zu HEVinfizierten Wildtieren und deren Ausscheidungen oder eine häufigere Beteiligung an Jagden die höhere Prävalenz in der Waldarbeitergruppe erklären.

Als ein Hauptübertragungsweg wird der Verzehr von Fleischprodukten, die von infizierten Tieren stammen, angesehen [6]. Dieser Weg wird auch direkt durch Ergebnisse von Fall- und Ausbruchsuntersuchungen, bei denen derselbe Virusstamm sowohl im Patienten als auch in Resten des verzehrten Lebensmittels nachgewiesen wurde, gestützt. So wurden aus Japan verschiedene Erkrankungsfälle nach dem Verzehr von nicht oder ungenügend erhitzter Leber oder Fleisch von Wildschweinen oder Rehwild aufgeklärt. In Frankreich wurde ein Hepatitis-E-Ausbruch mit 7 erkrankten Personen beschrieben, die eine kontaminierte traditionelle Wurst mit rohen Schweineleberanteilen gegessen hatten [15]. Auch aus der Schweiz ist ein ähnlicher Erkrankungsfall aufgeklärt worden, der nach dem Verzehr einer anderen Wurstart mit roher Schweineleber aufgetreten war [16]. Für Deutschland wurden verschiedene Fall-Kontroll-Studien durchgeführt, die auf den Verzehr von
Wild und Innereien [17], Schweineleber, Schweine- und Wildschweinfleisch [18] sowie Wurstprodukten [19] als höchsten Risikofaktor für eine Hepatitis-EErkrankung hinweisen.

Neben Fleischprodukten wurden verschiedentlich auch Hinweise auf andere Lebensmittel als mögliche Übertragungsvehikel gefunden [20]. Alternative Übertragungswege werden auch von Ernährungsstudien gestützt, in denen Vegetarier regelmäßig auch positive Antikörperbefunde tragen, wenngleich meistens mit deutlich geringerer Prävalenz als Omnivoren. HEV-RNA wurde auch an Beerenfrüchten und in Muscheln nachgewiesen. Für Deutschland gibt es hierzu bisher nur wenige Daten. Als Ursache für die HEV-RNA-Nachweise in diesen Lebensmittelarten werden unter anderem Kontaminationen mit Abwasser oder Dünger aus Schweinehaltungen angesehen.

Umweltkontaminationen mit Ausscheidungen von infizierten Schweinen, Wildtieren und Menschen werden auch als Ausgangspunkt für HEV-Infektionen diskutiert. Eine erste deutsche Studie hat beispielsweise in Abwasser und Flusswasser HEV-RNA nachweisen können [21]. Auch wenn deshalb Infektionen durch Aufnahme des kontaminierten Wassers möglich erscheinen, ist die relative Bedeutung dieses Übertragungsweges noch unklar.

Mensch-zu-Mensch-Übertragungen durch direkten Kontakt scheinen bei HEV und im Besonderen beim HEVGenotyp 3 kaum vorzukommen. Demgegenüber gelten Blut und Blutprodukte als mögliche Übertragungsvehikel. Studien in Deutschland konnten HEV-RNA in 1 von 4525 bis 1 von 597 der untersuchten Blutspenden nachweisen [22, 23]. Aufgrund des Risikos einer HEVÜbertragung durch Blutprodukte sind seit 2020 Blutspenden in Deutschland auf HEV zu untersuchen [24].

Weiterhin gelten Reisen in Länder, in denen die Genotypen 1 und 2 endemisch sind und aufgrund niedriger Hygienestandards über kontaminiertes Trinkwasser übertragen werden könnten, als Risiko für eine Infektion mit HEV. 
Tab. 3 Nachweis von HEV-RNA in Lebensmitteln vom Schwein, die in Deutschland bezogen wurden

\begin{tabular}{lll} 
Lebensmittel & $\begin{array}{l}\text { Nachweis von HEV-RNA } \\
\text { (Zahl positiver/Gesamtzahl untersuchter Proben) }\end{array}$ & Referenz \\
\hline Leber & $8 / 200(4,0 \%)$ & {$[45]$} \\
& $2 / 41(4,9 \%)$ & {$[46]$} \\
\hline Leberwurst & $11 / 50(22,0 \%)$ & {$[47]$} \\
& $5 / 40(12,5 \%)$ & {$[46]$} \\
Leberpaté & $6 / 40(15,0 \%)$ & {$[46]$} \\
Rohwurst & $14 / 70(20,0 \%)$ & {$[47]$} \\
(ohne Leber) & $0 / 10(0,0 \%)$ & {$[46]$}
\end{tabular}

\section{Bedeutung für die} Lebensmittelsicherheit

Wie oben bereits ausgeführt wurde, haben Fleisch und Fleischprodukte von Schweinen und Wildschweinen eine besondere Bedeutung für die Übertragung von $\mathrm{HEV}$ in Deutschland. In einigen Studien wurde untersucht, wie weit HEV-RNA in solchen Lebensmitteln in Deutschland verbreitet ist (• Tab. 3). Für Schweineleber aus dem Handel wurden hierbei Nachweisraten von 4,0-4,9\% gefunden. In Leberwürsten und -paté wurden 12,5-22,0\% RNA-positive Proben gefunden. Bei Rohwürsten ohne Leberanteil (z.B. Salami) ergab eine Studie etwa $20 \%$ positive Proben, während eine andere Studie keine RNA in solchen nachweisen konnte. Die Verwendung unterschiedlicher Methoden mit unterschiedlichen Nachweisgrenzen könnte die Diskrepanz zwischen beiden Studien erklären. Generell scheinen die höheren Nachweisraten für Wurstwaren im Vergleich zum Ausgangsmaterial (z. B. Leber) zunächst überraschend. Jedoch muss in Betracht gezogen werden, dass während der Herstellung meistens Fleisch und Organe mehrerer Tiere gemischt werden, wodurch sich die höheren Nachweisraten erklären lassen.

Bei der Interpretation der Nachweisraten ist generell problematisch, dass, methodisch bedingt, bisher nur RNA-Nachweise erfolgen können. Der Nachweis von infektiösen Viren direkt in Lebensmitteln, beispielsweise durch Anzucht in Zellkulturen, ist wegen des Fehlens entsprechender praktikabler und sensitiver Methoden derzeit noch nicht möglich. Weil aber die meisten Lebensmittel während ihrer Herstellung verschiedene phy- sikalisch-chemische Prozesse durchlaufen, könnte das enthaltene Virus währenddessen inaktiviert worden sein. Deshalb ist die Identifizierung von Risikolebensmitteln zurzeit noch schwierig und mit vielen Unsicherheiten behaftet.

Um Anhaltspunkte für die Stabilität von $\mathrm{HEV}$ in speziellen Lebensmitteln und unter spezifischen physikochemischen Bedingungen zu erhalten, wurden sowohl Inokulationsversuche mit Schweinen als auch Zellkulturuntersuchungen durchgeführt. So wurden unterschiedliche Lebensmittel verschiedenen Erhitzungsregimes unterzogen, danach durch intravenöse Injektion der aufgearbeiteten Proben in Schweine inokuliert und anschließend deren Serokonversion gemessen. Hierbei wurde gezeigt, dass eine Erhitzung von $71^{\circ} \mathrm{C}$ Kerntemperatur für $5 \mathrm{~min}$ bei Leber [25] und von $71^{\circ} \mathrm{C}$ für $20 \mathrm{~min}$ bei Leberpaté [26] zur HEV-Inaktivierung ausreichend war. In einer Zellkulturstudie führte die Erhitzung von $\mathrm{HEV}$ in Zellkulturmedium bei $70^{\circ} \mathrm{C}$ für $1 \mathrm{~min}$ $\mathrm{zu}$ einer 1000 -fachen Reduktion der Infektiosität, während bei $60^{\circ} \mathrm{C}$ für $1 \mathrm{~min}$ nur eine 10 -fache Reduktion erreicht wurde [27]. In diesem Zellkultursystem wurden auch andere Bedingungen genauer untersucht, wobei sich generell eine hohe Virusstabilität abzeichnete: Nur eine geringe Verminderung der Infektiosität ( $\leq 10$-fache Reduktion) wurde bei $\mathrm{pH}$-Werten zwischen 2 und 9 für $1 \mathrm{~h}$ [28], einer Salzkonzentration von $20 \%$ $\mathrm{NaCl}$ für 24h [29] oder einem hydrostatischen Druck von $200 \mathrm{MPa}$ für $1 \mathrm{~min}$ gefunden [30]. Starke Inaktivierungen (>1000-fache Reduktion) waren nur bei $\mathrm{pH} 1$ und 10 sowie bei $600 \mathrm{MPa}$ für 1 min festzustellen. Insgesamt deuten die Ergebnisse darauf hin, dass HEV in den meisten üblichen Lebensmitteln, die keiner starken Erhitzung oder hohen Drücken ausgesetzt sind, für lange Zeit infektiös bleiben kann.

\section{Weiterer Forschungsbedarf}

Aus den bisherigen Ausführungen kann weiterer Forschungsbedarf abgeleitet werden, um die Übertragung von HEV auf den Menschen und die dadurch ausgelösten Erkrankungsfälle $\mathrm{zu}$ verringern. Beim Übertragungsweg über tierische Lebensmittel gilt es zunächst, die Risikoprodukte, die hohe Mengen von infektiösem HEV enthalten können, weiter einzugrenzen. Dies könnte über detaillierte Untersuchungen bei einzelnen Erkrankungsfällen inklusive der Untersuchung von Lebensmittelresten oder über weitere Fall-Kontroll-Studien erfolgen. Auch die Untersuchung des Inaktivierungsverhaltens von HEV während der Herstellung verschiedener Fleischprodukte kann klären helfen, unter welchen Bedingungen HEV vollständig inaktiviert wird und welche Produkte somit kein Risiko darstellen. Hierzu wäre allerdings die vorherige Entwicklung von robust anwendbaren Methoden zur Infektiositätstestung von HEV in Lebensmitteln vonnöten. Wenn die entsprechenden Risikoprodukte identifiziert sind, könnten gezielte Verzehrempfehlungen besonders für Risikopatienten ein sinnvolles Instrument zur Verhinderung von Erkrankungen sein.

Weiterer Forschungsbedarfliegt in der Entwicklung von Strategien zur Minimierung des Kontaminationsrisikos von tierischen Lebensmitteln. Hierfür sollte die gesamte Lebensmittelkette, ausgehend von der Schweinehaltung, genauer untersucht werden. Beispielsweise könnte die Effizienz verschiedener Hygiene-, Haltungs- und Aufzuchtregimes zur Minimierung von HEV-Infektionen in Schweineherden analysiert werden. Auch die Möglichkeit von Impfungen in den Tierbeständen sollte hierbei genauer untersucht werden. Für die Schlachtung und Lebensmittelproduktion könnten verschiedene Hygienemaßnahmen auf ihre Anfälligkeit für Kreuzkontami- 
nationen mit HEV analysiert werden. Untersuchungen zur Wirksamkeit von Desinfektionsmitteln gegenüber $\mathrm{HEV}$, die auch mögliche Unterschiede zwischen unbehüllten HEV-Partikeln und solchen mit einer zusätzlichen Lipidhülle berücksichtigen, sind für die Entwicklung effizienter Desinfektionsregimes nötig. Außerdem wären Untersuchungen $\mathrm{zu}$ wirksamen Behandlungen von Lebensmitteln zur Reduzierung von HEV, zusätzlich zu Temperatur- und Hochdruckbehandlung, sinnvoll.

Wichtig wären auch weitergehende Untersuchungen $\mathrm{zu}$ alternativen Übertragungswegen. Besonders die Bedeutung von Umweltkontaminationen für die Übertragung von HEV wäre hier von Interesse. Dabei kämen sowohl den Kontaminationen von Gewässern, Übertragungen über Stäube oder aber Kontaminationen von pflanzlichen Lebensmitteln über Düngemittel oder die Bewässerung infrage. Auch hier ließen sich nach Vorliegen der Daten sicherlich effiziente Strategien zur Verhinderung solcher Kontaminationen erarbeiten.

\section{Fazit}

Die Zahl der gemeldeten Hepatitis-E-Fälle nahm in den letzten Jahren stark zu und chronische Infektionen bei Transplantationspatienten stellen eine relativ neue und ernst zu nehmende Problematik dar. In Deutschland kommt vor allem der zoonotische Genotyp 3 vor. Dieser ist in Haus- und Wildschweinen in Deutschland weitverbreitet und kann durch direkten Kontakt zu den Tieren oder durch den Verzehr daraus hergestellter Lebensmittel übertragen werden. $\mathrm{HEV}$ wird auch in verschiedenen Fleischprodukten in Deutschland nachgewiesen, wobei allerdings zumeist unklar ist, ob das nachgewiesene Virus noch infektiös ist oder während der Lebensmittelherstellung bereits inaktiviert wurde. Forschungsbedarf besteht weiterhin bei der Identifizierung von Risikolebensmitteln und bei Strategien zur Minimierung des Kontaminationsrisikos von Lebensmitteln, auch unter Berücksichtigung möglicher Übertragungswege in der Umwelt.

Das Bundesinstitut für Risikobewertung (BfR) hat verschiedene Empfeh- lungen erarbeitet, um das Risiko einer Ansteckung über die derzeit am besten untersuchten Übertragungswege $\mathrm{zu}$ minimieren [31]. So wird Jägern empfohlen, bei der Ausweidung von Wildschweinen generell Handschuhe zu tragen, um eine direkte Virusübertragung auszuschließen. Allgemein wird Verbrauchern empfohlen, Schweine- und Wildschweinfleisch sowie -leber vor dem Verzehr immer gut durchzuerhitzen, um eventuell enthaltenes Virus sicher $\mathrm{zu}$ inaktivieren. Für Risikogruppen wie Personen mit Lebervorschädigung oder Transplantationspatienten wird empfohlen, auf den Verzehr von kurzgereiften Rohwürsten zu verzichten, weil durch deren Herstellungsweise möglicherweise enthaltenes Virus wahrscheinlich nicht inaktiviert wird. Die Empfehlungen werden kontinuierlich angepasst, wenn neue belastbare Erkenntnisse zu Übertragungswegen, Risikolebensmitteln oder effizienten Inaktivierungsmethoden vorliegen.

\section{Korrespondenzadresse}

\section{Professor Dr. Reimar Johne}

Bundesinstitut für Risikobewertung

Max-Dohrn-Str. 8-10, 10589 Berlin,

Deutschland

reimar.johne@bfr.bund.de

Förderung. Reimar Johne dankt für die Förderung durch das Bundesministerium für Gesundheit (BMG; aufgrund eines Beschlusses des Deutschen Bundestags durch die Bundesregierung, Projekt CHED, Fördernummer ZMVI1-2518FSB705) und das Bundesministerium für Bildung und Forschung (BMBF; Projekt ZoRaHED, Förderkennzeichen 01KI2103).

Funding. Open Access funding enabled and organized by Projekt DEAL.

\section{Einhaltung ethischer Richtlinien}

Interessenkonflikt. R. Johne, N. Althof, K. Nöckler und A. Falkenhagen geben an, dass kein Interessenkonflikt besteht.

Für diesen Beitrag wurden von den Autoren keine Studien an Menschen oder Tieren durchgeführt. Für die aufgeführten Studien gelten die jeweils dort angegebenen ethischen Richtlinien.

Open Access. Dieser Artikel wird unter der Creative Commons Namensnennung 4.0 International Lizenz veröffentlicht, welche die Nutzung, Vervielfältigung, Bearbeitung, Verbreitung und Wiedergabe in jeglichem Medium und Format erlaubt, sofern Sie den/die ursprünglichen Autor(en) und die Quelle ordnungsge- mäß nennen, einen Link zur Creative Commons Lizenz beifügen und angeben, ob Änderungen vorgenommen wurden.

Die in diesem Artikel enthaltenen Bilder und sonstiges Drittmaterial unterliegen ebenfalls der genannten Creative Commons Lizenz, sofern sich aus der Abbildungslegende nichts anderes ergibt. Sofern das betreffende Material nicht unter der genannten Creative Commons Lizenz steht und die betreffende Handlung nicht nach gesetzlichen Vorschriften erlaubt ist, ist für die oben aufgeführten Weiterverwendungen des Materials die Einwilligung des jeweiligen Rechteinhabers einzuholen.

Weitere Details zur Lizenz entnehmen Sie bitte der Lizenzinformation auf http://creativecommons.org/ licenses/by/4.0/deed.de.

\section{Literatur}

1. Velavan TP, Pallerla SR, Johne R, Todt $D$, Steinmann E, Schemmerer M, Wenzel JJ, Hofmann J, Shih J, Wedemeyer H, BockCT(2021) HepatitisE: an update on one health and clinical medicine. Liver Int 41:1462-1473

2. Robert Koch-Institut (2021) SurvStat@RKI 2.0. https://survstat.rki.de.Zugegriffen: 8. Juli 2021

3. Faber M, Willrich N, Schemmerer M, Rauh C, KuhnertR,StarkK, WenzelJJ(2018) HepatitisEvirus seroprevalence, seroincidence and seroreversion in the German adult population. J Viral Hepat 25:752-758

4. Pallerla SR, Harms D, Johne R, Todt D, Steinmann E, Schemmerer M, Wenzel JJ, Hofmann J, Shih J, Wedemeyer H, Bock CT, Velavan TP (2020) Hepatitis E virus infection: circulation, molecular epidemiology, and impact on global health. Pathogens 9:856

5. Schemmerer M, Johne R, Erl M, Jilg W, Wenzel JJ (2019) Isolation of subtype 3c, 3e and 3f-like hepatitis E virus strains stably replicating to high viral loads in an optimized cell culture system. Viruses 11:483

6. Pavio N, Doceul V, Bagdassarian E, Johne R (2017) Recent knowledge on hepatitis $E$ virus in suidae reservoirs and transmission routes to human. Vet Res 48:78

7. Schielke A, Sachs K, Lierz M, Appel B, Jansen A, Johne R (2009) Detection of hepatitis Evirus in wild boars of rural and urban regions in Germany and whole genome characterization of an endemic strain. Virol J 6:58

8. Schielke A, Ibrahim V, Czogiel I, Faber M, SchraderC, Dremsek P, Ulrich RG, Johne R (2015) Hepatitis E virus antibody prevalence in hunters from a district in Central Germany, 2013: a cross-sectional study providing evidence for the benefit of protective gloves during disembowelling of wild boars. BMC Infect Dis 15:440

9. Spahr C, Knauf-Witzens T, Vahlenkamp T, Ulrich RG, Johne R (2018) Hepatitis Evirus and related viruses in wild, domestic and zoo animals: a review. Zoonoses Public Health 65:11-29

10. Anheyer-Behmenburg HE, Szabo K, Schotte U, Binder A, Klein G, Johne R (2017) Hepatitis E virus in wild boars and spillover infection in red and roe deer, Germany, 2013-2015. Emerg Infect Dis 23:130-133

11. Bächlein C, Becher P (2017) No evidence for zoonotic hepatitis $E$ virus infection through dairy milkin Germany. Hepatology 65:394-395 
12. Spahr C, Ryll R, Knauf-Witzens T, Vahlenkamp TW, Ulrich RG, Johne R (2017) Serological evidence of hepatitis $E$ virus infection in zoo animals and identification of a rodent-borne strain in a Syrian brown bear. Vet Microbiol 212:87-92

13. Krumbholz A, Mohn U, Lange J, Motz M, Wenzel JJ, Jilg W, Walther M, Straube E, Wutzler P, Zell R (2012) Prevalence of hepatitis E virus-specific antibodies in humans with occupational exposure to pigs. Med Microbiol Immunol 201:239-244

14. Dremsek P, Wenzel JJ, Johne R, Ziller M, Hofmann J, Groschup MH, Werdermann S, Mohn U, Dorn S, Motz M, Mertens M, Jilg W, Ulrich RG (2012) Seroprevalence study in forestry workers from eastern Germany using novel genotype 3- and rat hepatitis $E$ virus-specific immunoglobulin $G$ ELISAs. Med Microbiol Immunol 201:189-200

15. Colson P, Borentain P, Queyriaux B, Kaba M, Moal V, Gallian P, Heyries L, Raoult D, Gerolami R (2010) Pig liver sausage as a source of hepatitis $E$ virus transmission to humans. J Infect Dis 202:825-834

16. Kubacki J, Fraefel C, Jermini M, Giannini $P$, Martinetti G, Ripellino P, Bernasconi E, Sidler X, Stephan R, Bachofen C (2017) Complete genome sequences of two Swiss hepatitis $E$ virus isolates from human stool and raw pork sausage. Genome Announc 5:e00888-17

17. Wichmann O, Schimanski S, Koch J, Kohler M, Rothe C, Plentz A, Jilg W, Stark K (2008) Phylogenetic and case-control study on hepatitis $E$ virus infection in Germany. J Infect Dis 198:1732-1741

18. Faber M, Askar M, StarkK (2018) Case-control study on risk factors for acute hepatitis $E$ in Germany, 2012 to 2014. Euro Surveill 23:17-00469

19. Hriskova K, MarosevicD, Belting A, Wenzel JJ, Carl A, Katz K (2021) Epidemiology of hepatitis E in 2017 in Bavaria, Germany. Food Environ Virol. https://doi. org/10.1007/s12560-021-09474-0

20. Treagus S, Wright C, Baker-Austin C, Longdon B, Lowther J (2021) The foodborne transmission of hepatitis E virus to humans. Food Environ Virol 13:127-145

21. Beyer S, Szewzyk R, Gnirss R, Johne R, Selinka HC (2020) Detection and characterization of hepatitis Evirus genotype 3 in wastewater and urban surface waters in Germany. Food Environ Virol 12:137-147

22. Baylis SA, Gärtner T, Nick S, Ovemyr J, Blümel J (2012) Occurrence of hepatitis E virus RNA in plasma donations from Sweden, Germany and the United States. Vox Sang 103:89-90

23. Vollmer T, Diekmann J, Knabbe C, Dreier J (2019) Hepatitis E virus blood donor NAT screening: as much as possible or as much as needed? Transfusion 59:612-622

24. Paul-Ehrlich-Institut (2019) Bekanntmachung über die Zulassung von Arzneimitteln (Abwehr von Arzneimittelrisiken - Anordnung der Testung von Blutspendern zur Verhinderung einer Übertragung von Hepatitis-E-Virus durch Blutkomponenten zur Transfusion und Stammzellzubereitungen zur hämatopoetischen Rekonstitution. BundesAnzeigerv. 17.05.2019)

25. Feagins AR, Opriessnig T, Guenette DK, Halbur PG, Meng XJ (2008) Inactivation of infectious hepatitis E virus present in commercial pig livers sold in local grocery stores in the United States. Int J Food Microbiol 123:32-37

26. Barnaud E, Rogée S, Garry P, Rose N, Pavio N (2012) Thermal inactivation of infectious hepatitis Evirus in experimentally contaminated food. Appl Environ Microbiol 78:5153-5159

27. Johne R, Trojnar E, Filter M, Hofmann J (2016) Thermal stability of hepatitis $E$ virus as estimated by a cell culture method. Appl Environ Microbiol 82:4225-4231

28. Wolff A, Günther T, Albert T, Schilling-Loeffler $K$ Gadicherla AK, Johne R (2020) Stability of hepatitis E virus at different $\mathrm{pH}$ values. Int J Food Microbiol 325:108625

29. Wolff A, Günther T, Albert T, Johne R (2020) Effect of sodium chloride, sodium nitrite and sodium nitrate on the infectivity of hepatitis E virus. Food Environ Virol 12:350-354

30. Johne R, Wolff A, Gadicherla AK, Filter M, Schlüter O (2021) Stability of hepatitis E virus at high hydrostatic pressure processing. Int J Food Microbiol 339:109013

31. Bundesinstitut für Risikobewertung (2016) Fragen und Antworten zur Übertragung des Hepatitis E-Virus durch Wild- und Hausschweine und daraus gewonnene Lebensmittel. https://www. bfr.bund.de/cm/343/fragen-und-antworten-zuruebertragung-des-hepatitis-e-virus-durch-wildund-hausschweine-und-daraus-gewonnenelebensmittel.pdf.Zugegriffen: 8. Juli 2021

32. Kaci S, Nöckler K, Johne R (2008) Detection of hepatitis $E$ virus in archived German wild boar serum samples. Vet Microbiol 128:380-385

33. Adlhoch C, Wolf A, Meisel H, Kaiser M, Ellerbrok H, Pauli G (2009) High HEV presence in four different wild boar populations in East and West Germany. Vet Microbiol 139:270-278

34. Denzin N, Borgwardt J (2013) Vorkommen und geografische Verbreitung von Antikörpern gegen Hepatitis E-virus beim Wildschwein in SachsenAnhalt (2011). Berl Munch Tierarztl Wochenschr 126:230-235

35. Oliveira-Filho EF, Bank-Wolf BR, Thiel HJ, König M (2014) Phylogenetic analysis of hepatitis E virus in domestic swine and wild boar in Germany. Vet Microbiol 174:233-238

36. Vina-Rodriguez A, Schlosser J, Becher D, Kaden V, Groschup MH, Eiden M (2015) Hepatitis E virus genotype 3 diversity: phylogenetic analysis and presence of subtype $3 \mathrm{~b}$ in wild boar in Europe. Viruses 7:2704-2726

37. Weigand K, Weigand K, Schemmerer M, Müller M, Wenzel JJ (2018) Hepatitis E seroprevalence and Genotyping in a cohort of wild boars in southern Germany and eastern Alsace. Food Environ Virol 10:167-175

38. Bächlein C, Schielke A, Johne R, Ulrich RG, Baumgaertner W, Grummer B (2009) Prevalence of Hepatitis $E$ virus-specific antibodies in sera of German domestic pigs estimated by using different assays. Vet Microbiol 144:187-191

39. Wacheck S, Werres C, Mohn U, Dorn S, SoutschekE, Fredriksson-Ahomaa M, Märtlbauer E (2012) Detection of $\lg M$ and $\lg G$ against hepatitis $E$ virus in serum and meat juice samples from pigs at slaughter in Bavaria, Germany. Foodborne Pathog Dis 9:655-660

40. Bächlein C, Seehusen $F$, Nathues $H$, grosse Beilage E, Baumgärtner W, Grummer B (2013) Molecular detection of hepatitis Evirus in German domestic pigs. Berl Munch Tierarztl Wochenschr 126:25-31

41. Krumbholz A, Joel $S$, Neubert A, Dremsek $P$, Dürrwald R, Johne R, Hlinak A, Walther M, Lange J, Wutzler P, Sauerbrei A, Ulrich RG, Zell R (2013) Agerelated and regional differences in the prevalence of hepatitis E virus-specific antibodies in pigs in Germany. Vet Microbiol 167:394-402

42. Dremsek P, Joel S, Baechlein C, Pavio N, Schielke A, Ziller M, Dürrwald R, Renner C, Groschup $M H$, Johne R, Krumbholz A, Ulrich RG (2013) Hepatitis E virus seroprevalence of domestic pigs in Germany determined by a novel in-house and two reference ELISAs. JVirol Methods 190:11-16

43. Machnowska P, Ellerbroek L, Johne R (2014) Detection and characterization of potentially zoonotic viruses in faeces of pigs at slaughter in Germany. Vet Microbiol 168:60-68

44. Dzierzon J, Oswaldi V, Merle R, Langkabel N, Meemken D (2020) High predictive power of meat juice serology on the presence of hepatitis Evirus in slaughter pigs. Foodborne Pathog Dis 17:687-692

45. Wenzel JJ, Preiss J, Schemmerer M, Huber B, Plentz A, Jilg W (2011) Detection of hepatitis E virus (HEV) from porcine livers in Southeastern Germany and high sequence homology to human HEV isolates. J Clin Virol 52:50-54

46. Pallerla SR, Schembecker S, Meyer CG, Linh LTK, Johne R, Wedemeyer $\mathrm{H}$, Bock CT, Kremsner PG, Velavan TP (2021) Hepatitis E virus genome detection in commercial pork livers and pork meat products in Germany. J Viral Hepat 28:196-204

47. Szabo K, Trojnar E, Anheyer-Behmenburg H, Binder A, Schotte U, Ellerbroek L, Klein G, Johne R (2015) Detection of hepatitis E virus RNA in raw sausages and liver sausages from retail in Germany using an optimized method. Int J Food Microbiol 215:149-156 